\title{
CONSENSUS-BASED DISTRIBUTED UNSCENTED PARTICLE FILTER
}

\author{
Arash Mohammadi and Amir Asif \\ Department of Computer Science and Engineering \\ York University, Toronto, ON, Canada M3J 1P3 \\ Email:\{marash, asif\}@cse.yorku.ca
}

\begin{abstract}
In this paper, we propose a consensus-based, distributed implementation of the unscented particle filter (CD/UPF) that extends the distributed Kalman filtering framework to non-linear, distributed dynamical systems with non-Gaussian excitations. Compared to the existing distributed implementations of the particle filter, the CD/UPF offers two advantages. First, it uses all available local observations including the most recent ones in deriving the proposal distribution. Second, computation of global estimates from local estimates during the consensus step is based on an optimal fusion rule. In our bearingonly tracking simulations, the performance of the proposed CD/UPF is virtually indistinguishable from its centralized counterpart.
\end{abstract}

Index Terms - Distributed estimation, Unscented Particle Filter, Consensus Algorithm, Data Fusion, Non-linear Estimation.

\section{INTRODUCTION}

The paper focuses on distributed tracking algorithms for non-linear, non-Gaussian data fusion problems in sensor networks. Of particular interest are state estimation and tracking applications based on observation nodes configured as a distributed network (one that does not need a central processing unit) for surveillance and condition monitoring in large-scale systems with non-linear dynamics, such as multirobot systems and cooperating, unmanned aerial vehicles (UAV).

Estimation in sensor networks is typically centralized, where the sensor nodes communicate their raw observations either directly or via a multi-hop relay to a central processing unit, referred to as the fusion centre (FC), which is responsible for performing the estimation task. Such centralized approaches are generally unscalable and also susceptible to failure in case the FC breaks down. Further, energy consumption, especially in multi-hop relay communication, is uneven with nodes closer to the FC requiring more energy because of a higher number of information transfers. With a change in the network topology, the routing tables need to be redesigned in centralized estimation adding to the complexity of the system. The alternative to the centralized framework is distributed estimation, which does not require a fusion centre and, therefore, offers greater immunity to network failure. The sensor nodes exchange data only with their neighboring nodes and do not require any prior knowledge of the network topology. Because of the aforementioned advantages, there has been a surge of interest in distributed estimation algorithms. Broadly speaking, the distributed estimation approaches fall in two categories: (i) Message passing schemes where information flows in a sequential manner from a node to one of its neighboring nodes [1] till the entire network is traversed, and; (ii) Diffusive schemes where each node diffuses its local information throughout the network by communicating with its neighboring nodes. No routing table needs to be constructed. Category (ii) includes average consensus-based algorithms [2]-[4] and has the added advantage of being robust to changes in the network topology. This, however, comes at the expense of an increased communications overhead.

Among the consensus-based distributed approached, distributed Kalman filters [2, 5] for linear systems have been widely explored. In comparison, the consensus-based distributed particle filters for nonlinear systems are less explored. Majority of such particle filter implementations use Gaussian approximations $[3,4]$ for the local posteriors, which are then used to derive the global posterior using a suboptimal fusion rule. In this work, we instead focus on a distributed implementation of the unscented particle filter (UPF), which couples the unscented Kalman filter (UKF) with the particle filter such that the UKF provides a Gaussian approximation to the posterior distribution, which is then used as the proposal distribution [6] in the PF. The resulting algorithm is referred to as the consensus-based, distributed implementation of the unscented particle filter (CD/UPF), which offers two significant improvements to the existing distributed framework for the particle filter: (i) Unlike the current implementations [4] of the particle filter, the proposed CD/UPF uses available local observations (including the most recently acquired ones) to derive the proposal distribution, and; (ii) Computation of global estimates from local estimates during the consensus step is based on an optimal fusion rule. Further, the CD/UPF paves the way for incorporating future developments in consensus-based distributed Kalman filters to be used in the distributed particle filtering framework. The rest of paper is organized as follows. In Section 2, the centralized $\mathrm{UPF}$ is reviewed. The proposed CD/UPF is presented in Section 3. Section 4 illustrates the effectiveness of the CD/UPF in tracking applications through simulations. Section 5 concludes the paper.

\section{CENTRALIZED UNSCENTED PARTICLE FILTER}

Consider a network comprising of $N$ nodes observing the state vector $\mathbf{x}=\left[X_{1}, \ldots, X_{n_{x}}\right]^{T}$ with dimensions $n_{x}$. Node $l,(1 \leq l \leq N)$, makes measurements $\mathbf{z}^{(l)}(k)$ at time instants $k,(1 \leq k)$ such that

State Model: $\quad \mathbf{x}(k)=f(\mathbf{x}(k-1))+\boldsymbol{\xi}(k-1)$

Observation Model:

$$
\underbrace{\left[\begin{array}{c}
\mathbf{z}^{(1)}(k) \\
\vdots \\
\mathbf{z}^{(N)}(k)
\end{array}\right]}_{\mathbf{z}(k)}=\underbrace{\left[\begin{array}{c}
\boldsymbol{g}^{(1)}(\mathbf{x}(k)) \\
\vdots \\
\boldsymbol{g}^{(N)}(\mathbf{x}(k))
\end{array}\right]}_{g(\mathbf{x}(k))}+\underbrace{\left[\begin{array}{c}
\boldsymbol{\zeta}^{(1)}(k) \\
\vdots \\
\boldsymbol{\zeta}^{(N)}(k)
\end{array}\right]}_{\boldsymbol{\zeta}(k)}
$$

where $\{\boldsymbol{\xi}(\cdot), \boldsymbol{\zeta}(\cdot)\}$ are, respectively, the global uncertainties in the process and observation models. Unlike the Kalman filter, the state and observation functions $\{f(\cdot), g(\cdot)\}$ can possibly be nonlinear, 
and vectors $\{\boldsymbol{\xi}(\cdot), \boldsymbol{\zeta}(\cdot)\}$ are not necessarily white Gaussian. The nodes of the network are modeled as vertices of the graph $\mathcal{G}=$ $\{\boldsymbol{\nu}, \mathcal{E}\}$, namely as elements of the node set $\boldsymbol{\nu}=\{1, \ldots, N\}$. The edge set $\mathcal{E} \subseteq(\boldsymbol{\nu} \times \boldsymbol{\nu})$ represents the network's communication constraints, i.e., if node $i$ can send information to node $j$ then $(i, j) \in \mathcal{E}$. For graph $\mathcal{G}$, the maximum degree $\Delta_{\mathcal{G}}=\max _{l} D^{(l)}$, where $D^{(l)}$ is the number of neighboring nodes for node $l$.

2.1. Centralized Particle Filter: is based on the principle of sequential importance sampling (SIS) [6], a suboptimal technique for recursive Bayesian estimation through Monte Carlo simulations. Importance sampling is an approach to evaluate an integral, e.g.,

$$
\mathcal{E}\{h(\mathbf{x})\}=\int h(\mathbf{x}) p(\mathbf{x} \mid \mathbf{z}) d \mathbf{x}=\int h(\mathbf{x}) \frac{p(\mathbf{x} \mid \mathbf{z})}{q(\mathbf{x} \mid \mathbf{z})} q(\mathbf{x} \mid \mathbf{z}) d \mathbf{z},
$$

where $\mathcal{E}$ denotes expectation. A numeric way to compute $\mathcal{E}\{h(\mathbf{x}(k))\}$ is to draw $N_{s}$ random samples (vector particles) $\left\{\mathbb{X}_{i}(k)\right\}_{i=1}^{N_{s}}$ from the probability distribution $p(\mathbf{x}(k) \mid \mathbf{z}(k))$, evaluate the function $h(\mathbf{x}(k))$ at these samples, and then compute their statistical mean. In practice, the posterior $p(\mathbf{x}(k) \mid \mathbf{z}(k))$ is not known, therefore, the particles are instead derived from a proposal distribution $q(\mathbf{x}(k) \mid \mathbf{z}(k))$ with normalized weights $W_{i}(k)$, for $\left(1 \leq i \leq N_{s}\right)$, associated to the vector particles. Assuming that the evolution of the state variables is Markovian, the weights are updated as follows

$$
W(k)=W(k-1) \frac{p(\mathbf{z}(k) \mid \mathbf{x}(k)) p(\mathbf{x}(k) \mid \mathbf{x}(k-1))}{q(\mathbf{x}(k) \mid \mathbf{x}(0: k-1), \mathbf{z}(1: k))},
$$

where $\mathbf{z}(1: k)$ includes all observations $\mathbf{z}$ from iteration 1 to $k$. A similar notation is used for the remaining variables. It has been proved [6] that the optimal choice for the proposal distribution is

$q(\mathbf{x}(k) \mid \mathbf{x}(0: k-1), \mathbf{z}(1: k))=p(\mathbf{x}(k) \mid \mathbf{x}(0: k-1), \mathbf{z}(1: k))$.

Because of the difficulty in sampling Eq. (5), a common choice [6] for the proposal distribution is the transition pdf, $p(\mathbf{x}(k) \mid \mathbf{x}(k-1))$, where the weights are pointwise evaluation of the likelihood function at the particle values. Such an approach is suboptimal as it does not depend on the newly acquired observations and leads to degeneracy with a few samples having relatively higher weights. Next, we describe the UPF, which approximates the proposal distribution with a Gaussian pdf and takes into account the most recent observations.

2.2. Centralized Unscented Particle Filter: The unscented particle filter (UPF) couples the particle filter with the unscented Kalman filter (UKF) based on the configuration shown in Fig. 1. In the UPF, the optimal proposal distribution function (Eq.(5)) is approximated as a Gaussian whose statistics (mean and error covariance matrix) are computed using the UKF. The UPF consists of the following steps. Let $\left\{W_{i}(k), \mathbb{X}_{i}(k)\right\}_{i=1}^{N_{s}}$ be the set of particles and their associated weights at the end of iteration $k$ of the particle filter in steady state.

1. Calculate the empirical mean and covariance of the particles.

$$
\begin{aligned}
\tilde{\mathbf{x}}(k) & =\sum_{i=1}^{N_{s}} W_{i}(k) \mathbb{X}_{i}(k) \\
\tilde{\boldsymbol{P}}(k) & =\sum_{i=1}^{N_{s}} W_{i}(k)\left(\mathbb{X}_{i}(k)-\overline{\mathbf{x}}(k)\right)\left(\mathbb{X}_{i}(k)-\overline{\mathbf{x}}(k)\right)^{T}
\end{aligned}
$$

2. Calculate a set of $\left(2 n_{x}+1\right)$ deterministic samples (sigma points) $\mathcal{S}=\left\{\mathcal{W}_{i}, \chi_{i}(k)\right\}_{i=0}^{2 n_{x}}$ based on the following scheme

$$
\chi_{i}(k)=\tilde{\mathbf{x}}(k) \pm\left\{\sqrt{\left(n_{x}+\kappa\right) \tilde{\boldsymbol{P}}(k)}\right\}_{i}
$$

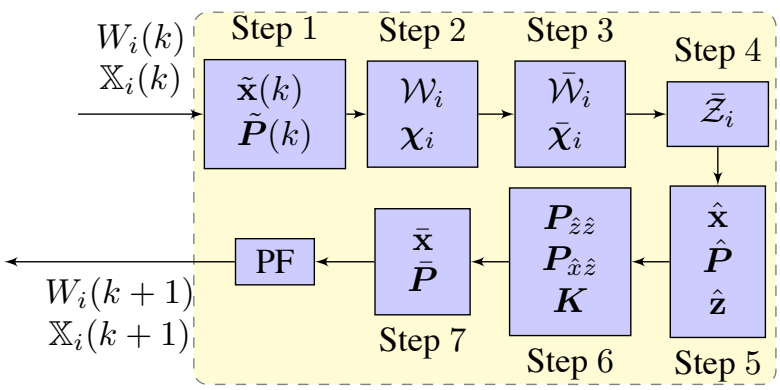

Fig. 1. Block diagram representing iteration $k+1$ of the UPF. Steps 1-7 are described in Section 2. Variables with a hat, e.g., $\hat{\mathbf{x}}$, are estimated at time index $(k+1 \mid k)$ and variables with a bar, e.g., $\overline{\mathbf{x}}(k)$, are estimated at index $(k \mid k)$

where term $\left\{\sqrt{\left(n_{x}+\kappa\right) \tilde{\boldsymbol{P}}(k)}\right\}_{i}$ corresponds to the $i^{\text {th }}$ column of the square root of matrix $\left(n_{x}+\kappa\right) \tilde{\boldsymbol{P}}(k)$ and the initial condition is given by $\chi_{0}(k)=\tilde{\mathbf{x}}(k)$. The corresponding weights for the Sigma points $\left\{\mathcal{W}_{i}\right\}_{i=1}^{2 n_{x}}$ are given by $\mathcal{W}_{i}=$ $1 /\left(2\left(n_{x}+\kappa\right)\right)$, where $\kappa$ is a scaling parameter and the initial condition for the sigma points is $\mathcal{W}_{0}=\kappa /\left(n_{x}+\kappa\right)$.

3. Propagate the sigma points computed in Step 2 through the state function to generate the predicted sigma points

$$
\chi_{i}(k+1 \mid k)=f\left(\chi_{i}(k)\right), \quad \text { for } i=0, \ldots, 2 n_{x} .
$$

4. Propagate $\chi_{i}(k+1 \mid k)$ through the measurement function to generate the predicted observation sigma points

$$
\mathcal{Z}_{i}(k+1 \mid k)=g\left(\chi_{i}(k+1 \mid k)\right), \quad \text { for } i=0, \ldots, 2 n_{x} .
$$

5. Estimate the predicted sigma points estimate $\mathbf{x}(k+1 \mid k)$, error covariance matrix $\boldsymbol{P}(k+1 \mid k)$, and the predicted $\mathbf{z}(k+1 \mid k)$.

$$
\begin{aligned}
\mathbf{x}(k+1 \mid k) & =\sum_{i=0}^{2 n_{x}} \mathcal{W}_{i} \boldsymbol{\chi}_{i}(k+1 \mid k) \\
\boldsymbol{P}(k+1 \mid k) & =\sum_{i=0}^{2 n_{x}} \mathcal{W}_{i}\left(\chi_{i}(k+1 \mid k)-\mathbf{x}(k+1 \mid k)\right) \\
& \times\left(\chi_{i}(k+1 \mid k)-\mathbf{x}(k+1 \mid k)\right)^{T} \\
\mathbf{z}(k+1 \mid k) & =\sum_{i=0}^{2 n_{x}} \mathcal{W}_{i} \mathcal{Z}_{i}(k+1 \mid k)
\end{aligned}
$$

6. Next, the autocovariance $\boldsymbol{P}_{z z}(k+1 \mid k)$ of predicted observations, the cross-covariance $\boldsymbol{P}_{x z}(k+1 \mid k)$ between predicted observation and predicted state estimates are computed. The respective expressions are similar to Eq. (12).

7. Finally, estimate the statistics of the proposal distribution.

$$
\begin{aligned}
& \overline{\mathbf{x}}(k+1)=\mathbf{x}(k+1 \mid k)+\boldsymbol{K}(\mathbf{z}(k+1)-\mathbf{z}(k+1 \mid k))(14) \\
& \overline{\boldsymbol{P}}(k+1)=\boldsymbol{P}(k+1 \mid k)-\boldsymbol{K} \boldsymbol{P}_{z z}(k+1 \mid k) \boldsymbol{K}^{T}, \\
& \text { where the Kalman gain } \boldsymbol{K}=\boldsymbol{P}_{x z}(k+1 \mid k) \boldsymbol{P}_{z z}(k+1 \mid k)^{-1} .
\end{aligned}
$$

After executing Steps 1-7, the UPF proceeds with the next iteration of the particle filters. In the UPF algorithm, Steps 1-6 are performed off-line and the new measurements are only involved in Step 7. 


\section{CONSENSUS-BASED DISTRIBUTED UPF}

In distributed estimation, the local particles and their weights are based only on the local observations $\mathbf{z}^{(l)}(k+1)$ resulting in inconsistent state estimates $\mathcal{E}\left(\mathbf{x}(k+1) \mid z^{(l)}(k+1)\right)$ across the network. A consensus step (Section 3.1) is introduced to provide consistency in the local estimates. As in [3, 4], we model the local posterior with a Gaussian but develop consensus-based distributed counterpart of the optimal decentralized fusion rule [8]. We go one step further and use such Gaussian approximations in the context of local UPF as the proposal distribution. Below, we outline the main steps followed at each node $(1 \leq l \leq N)$ of the CD/UPF and the consensus approach. The filter is assumed to be in steady state and at iteration $k$.

1. At time instant $k$, all nodes are assumed to have reached a consensus with a single set of global estimates $\overline{\mathbf{x}}_{\text {global }}(k \mid k)$ and $\overline{\boldsymbol{P}}_{\text {global }}(k \mid k)$ across the network. A new measurement $\mathbf{z}^{(l)}(k+1)$ is now available at the local nodes. Node $l$ generates the Sigma points $\left\{\chi_{i}^{(l)}(k)\right\}_{i=1}^{2 n_{x}+1}$ by substituting $\overline{\mathbf{x}}_{\text {global }}(k \mid k)$ and $\overline{\boldsymbol{P}}_{\text {global }}(k \mid k)$ in Eq. (8).

2. Node $l$ computes the statistics of its local proposal distribution $\left(\overline{\mathbf{x}}^{(l)}(k+1), \overline{\boldsymbol{P}}^{(l)}(k+1)\right)$ using Eqs. (9)-(15).

3 . Node $l$ generate $N_{s}$ random particles $\mathbb{X}_{i}^{(l)}(k+1)$ from its proposal distribution and computes their corespondent weights $W_{i}^{(l)}(k+1)$ based on Eq. (4). After this step, node $l$ has a set of particles and their associated weights that approximate the local posterior distribution $p\left(\mathbf{x}(k+1) \mid \mathbf{z}^{(l)}(k+1)\right)$.

4. Based on Eqs. (6) and (7), node $l$ computes the MMSE estimate $\overline{\mathbf{x}}^{(l)}(k+1 \mid k+1)$ and its corresponding error covariance $\overline{\boldsymbol{P}}^{(l)}(k+1 \mid k+1)$ (local statistics) of the state variables.

5. The final step of the CD/UPF algorithm is the consensus step used to compute a consistent set of values for the global statistics $\overline{\mathbf{x}}_{\text {global }}(k+1 \mid k+1)$ and $\overline{\boldsymbol{P}}_{\text {global }}(k+1 \mid k+1)$ at time $k+1$ from the local statistics, which is covered in Section 3.1.

3.1. Consensus Step: In synchronous average consensus algorithms [2], each node communicates with all its neighbors and updates its current consensus state at each consensus iteration $t$. The CD/UPF algorithm uses the following fusion rules to fuse local statistics $\left\{\overline{\mathbf{x}}^{(l)}(k+1 \mid k+1), \overline{\boldsymbol{P}}^{(l)}(k+1 \mid k+1)\right\}_{l=1}^{N}$ into a common set of global statistics denoted by $\overline{\mathbf{x}}_{\text {global }}(k+1 \mid k+1)$ and $\overline{\boldsymbol{P}}_{\text {global }}(k+1 \mid k+1)$.

$$
\begin{aligned}
& \overline{\boldsymbol{P}}_{\begin{array}{c}
(k+1 \mid k+1) \\
\text { global }
\end{array}}^{(l)^{-1}}=\boldsymbol{P}_{(k+1 \mid k)}^{(l)-1}+\underbrace{\sum_{j=1}^{N}\left\{\overline{\boldsymbol{P}}_{(k+1 \mid k+1)}^{(j)^{-1}}-\boldsymbol{P}_{(k+1 \mid k)}^{(j)^{-1}}\right\}}_{\boldsymbol{P}_{c}(\infty)} \\
& \overline{\mathbf{x}}_{\substack{(k+1 \mid k+1) \\
\text { global }}}^{(l)}=\overline{\boldsymbol{P}}_{\substack{(k+1 \mid k+1) \\
\text { global }}}^{(l)^{-1}}\left[\boldsymbol{P}_{(k+1 \mid k)}^{(l)^{-1}} \mathbf{x}_{(k+1 \mid k)}^{(l)}\right. \\
& +\underbrace{\left.\sum_{j=1}^{N}\left\{\boldsymbol{P}_{(k+1 \mid k+1)}^{(j)^{-1}} \mathbf{x}_{(k+1 \mid k+1)}^{(j)}-\boldsymbol{P}_{(k+1 \mid k)}^{(j)^{-1}} \mathbf{x}_{(k+1 \mid k)}^{(j)}\right\}\right]}_{\mathbf{x}_{c}(\infty)},
\end{aligned}
$$

where the time indices are shown as subscripts to save on space. In Eqs. (16) and (17), $\left\{\mathbf{x}_{c}(\infty), \boldsymbol{P}_{c}(\infty)\right\}$ are obtained by iterating the following average consensus equations where $\epsilon \in\left(0,1 / \Delta_{\mathcal{G}}\right)$ [2].

$$
\begin{aligned}
\mathbf{x}_{c}^{(l)}(t+1) & =\mathbf{x}_{c}^{(l)}(t)+\epsilon \sum_{j \in \mathcal{K}^{(l)}}\left(\mathbf{x}_{c}^{(j)}(t)-\mathbf{x}_{c}^{(l)}(t)\right) \\
\boldsymbol{P}_{c}^{(l)}(t+1) & =\boldsymbol{P}_{c}^{(l)}(t)+\epsilon \sum_{j \in \aleph^{(l)}}\left(\boldsymbol{P}_{c}^{(j)}(t)-\boldsymbol{P}_{c}^{(l)}(t)\right) .
\end{aligned}
$$

Table 1. Comparison of the computational complexity for the centralized UPF versus the proposed CD/UPF.

\begin{tabular}{|c|c|c|c|c|}
\hline & \multicolumn{2}{|c|}{$\begin{array}{c}\text { UKF } \\
\text { Complexity }\end{array}$} & $\begin{array}{c}\text { PF } \\
\text { Complexity }\end{array}$ & $\begin{array}{c}\text { Consensus } \\
\text { step }\end{array}$ \\
\hline \multirow{2}{*}{$\mathrm{UPF}$} & $\begin{array}{l}\max \left(\mathrm{O}\left(n_{x}^{3}\right),\right. \\
\left.\mathrm{O}\left(N^{3}\right), \mathrm{O}\left(n_{x} N^{2}\right)\right)\end{array}$ & $\mathrm{O}\left(n_{x}^{2}+N^{2}\right) N_{s}$ & - \\
\hline $\mathrm{CD} /$ & Per node & $\mathrm{O}\left(n_{x}^{3}\right)$ & $\mathrm{O}\left(N_{s} n_{x}^{2}\right)$ & $\mathrm{O}\left(n_{x} \Delta_{\mathcal{G}} N_{c}\right)$ \\
\cline { 2 - 5 } $\mathrm{UPF}$ & Total & $\mathrm{O}\left(N n_{x}^{3}\right)$ & $\mathrm{O}\left(N N_{s} n_{x}^{2}\right)$ & $\mathrm{O}\left(N n_{x} \Delta_{\mathcal{G}} N_{c}\right)$ \\
\hline
\end{tabular}

till they converge to $\left\{\mathbf{x}_{c}(\infty), \boldsymbol{P}_{c}(\infty)\right\}$. The initial conditions are

$$
\begin{aligned}
\boldsymbol{P}_{c}^{(l)}(t=0) & =\overline{\boldsymbol{P}}_{(k+1 \mid k+1)}^{(j)^{-1}}-\boldsymbol{P}_{(k+1 \mid k)}^{(j)^{-1}} \\
\mathbf{x}_{c}^{(l)}(t=0) & =\boldsymbol{P}_{(k+1 \mid k+1)}^{(j)^{-1}} \mathbf{x}_{(k+1 \mid k+1)}^{(j)}-\boldsymbol{P}_{(k+1 \mid k)}^{(j)^{-1}} \mathbf{x}_{(k+1 \mid k)}^{(j)} .
\end{aligned}
$$

Note that the consensus approach in Eqs. (18)-(19) is a distributed algorithm where each node communicates only with its neighboring nodes. Convergence of the consensus algorithms has been extensively studied $[2,5]$, e.g., reference [5] has shown that achieving consensus in a finite number of iterations is possible. As studied in $[3,4]$, we assume that consensus is reached between two successive observations. Such an assumption is reasonable in applications where communication is relatively inexpensive as compared to sensing, e.g., in rendezvous control or coordination of mobile sensors.

3.2 Computational Complexity: Table 1 compares the computational complexity of the centralized implementation of the UPF versus its distributed counterpart. The CD/UPF introduces an additional consensus step, whose complexity is derived as a function of the maximum degree $\Delta_{\mathcal{G}}$ of the network and the total number of consensus iterations $N_{c}$ required to reach a global consensus. Due to lack of space, we omit the derivation here but observe that for $\left(n_{x}<<N\right)$, the computational complexities of the CD/UPF and centralized UPF are comparable except that in the $\mathrm{CD} / \mathrm{UPF}$, the computations are distributed over the entire network. On the other hand, the FC in the centralized UPF performs most of the computational work.

We also note that for $N$ sensor nodes, each with at most $\Delta_{\mathcal{G}}$ neighbors, and for $N_{c}$ consensus iterations, the number of message transfers in the $\mathrm{CD} / \mathrm{UPF}$ is $\mathrm{O}\left(N \Delta_{\mathcal{G}} N_{c}\right)$.

\section{EXPERIMENTAL RESULTS}

A distributed bearing-only target tracking application [7] is simulated to test the proposed $\mathrm{CD} / \mathrm{UPF}$ algorithm, where the trajectory (position $[X, Y]$ and velocity $[\dot{X}, \dot{Y}]$ ) of a maneuvering target is estimated. For such applications, a multiple mode approach [7] using a combination of: (i) Constant velocity (CV) model; (ii) Clockwise coordinated turn (CT) model; (iii) Anticlockwise CT model, and; (iv) Constant acceleration model is used to simulate the target motion. Following [3,4], we use a single motion CV model with process noise of known statistical distribution in our simulations. A sensor network of $N=20$ nodes is considered where sensors are distributed randomly in a square region. Each sensor communicates only with sensors within its connectivity radius of $\sqrt{2 \log (N) / N}$ unit. Measurements are the target's bearings with respect to the platform of each node referenced (clockwise positive) to the $y$-axis, i.e.,

$$
Z^{(l)}(k)=\arctan \left(\frac{X(k)-X^{(l)}}{Y(k)-Y^{(l)}}\right)+\omega^{(l)}(k),
$$

where $\left(X^{(l)}, Y^{(l)}\right)$ are the coordinates of node $l$. In this paper, the observation noise model is assumed to be state dependent such that 


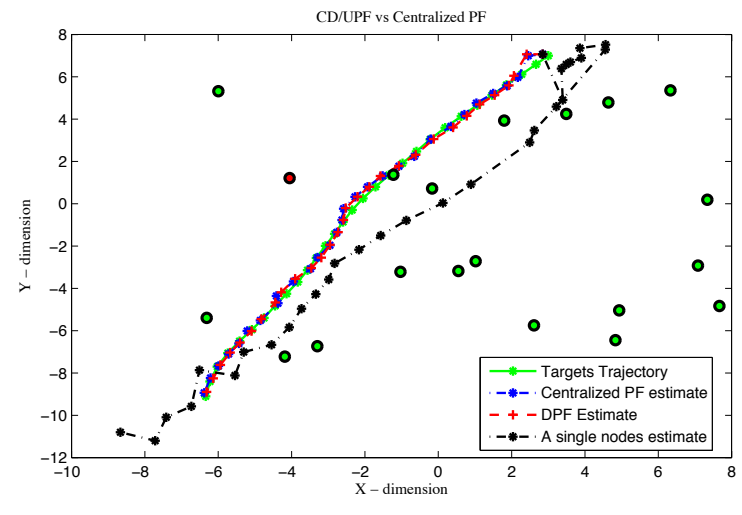

Fig. 2. Target's track estimated using the centralized UPF, CD/UPF, and stand-alone scenario from a single node.

the bearing noise variance $\sigma^{(l)^{2}}\left(r^{(l)}(k)\right)$ at node $l$ depends on the distance $r^{(l)}(k)$ between the observer and target, i.e.,

$$
\sigma^{(l)^{2}}\left(r^{(l)}(k)\right)=0.08 r^{(l)^{2}}(k)+0.1150 r^{(l)}(k)+1.7405 .
$$

To quantify the tracking performance of the proposed CD/UPF, three scenarios were considered: (i) Centralized scenario where each node has access to the local likelihoods of all other nodes. We use the performance of the centralized UPF as the base performance; (ii) Distributed scenario using the CD/UPF, and; (iii) Stand-alone scenario where no communication of likelihood or estimate occurs between the neighboring nodes. Target starts its track from coordinates $(3,7)$ units, with the initial course set at $-140^{\circ}$. Initialization of the filter is done according to [7] with $\sigma_{v}=0.16$. Each node uses $N_{s}=1000$ particles to approximate the posterior distribution. Sensors are randomly placed in an inner square of $(8 \times 8)$ units. One realization of the sensor placement is shown in Fig. 2, which also shows the target's track together with the outputs of the centralized, $\mathrm{CD} / \mathrm{UPF}$, and stand-alone estimation algorithms. It is obvious that the single node looses the track while the centralized and the $\mathrm{CD} / \mathrm{UPF}$ do a reasonable job in tracking the target. The difference between the centralized and CD/UPF implementation is almost negligible. Based on a 1000-run Monte-Carlo simulation, Fig. 3 shows the root mean square (RMS) error curves for the target's position versus time. The RMS error is significantly low for the centralized and $\mathrm{CD} / \mathrm{UPF}$ showing that the distributed $\mathrm{CD} / \mathrm{UPF}$ is a near optimal implementation of its centralized version. The stand alone implementation gives the largest error. Beside error analysis, statistical measures are also used to evaluate the performance of the CD/UPF algorithm. Fig. 4 compares the empirical cumulative density functions (CDF) obtained from the centralized and distributed posteriors at four different iterations. The CDFs are almost identical. One and two dimensional 2-sample Kolmogorov-Smirnov (KS) test, which compares the distributions of the values in the two data vectors is also conducted. The null hypothesis in the KS-test is that data samples are from the same continuous distribution. Our simulation results mostly accept the null hypothesis at the 0.05 significance level.

\section{SUMMARY}

In this paper, we proposed a consensus-based distributed implementation of the unscented particle filter (CD/UPF). The CD/UPF extends consensus-based distributed Kalman filtering framework to

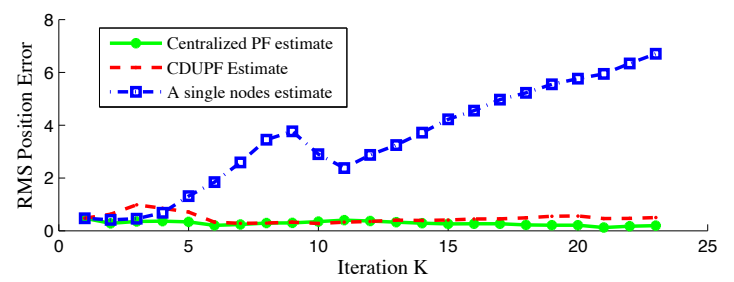

Fig. 3. RMS errors in the estimated target position from the centralized UPF, CD/UPF, and stand-alone case as a function of time.
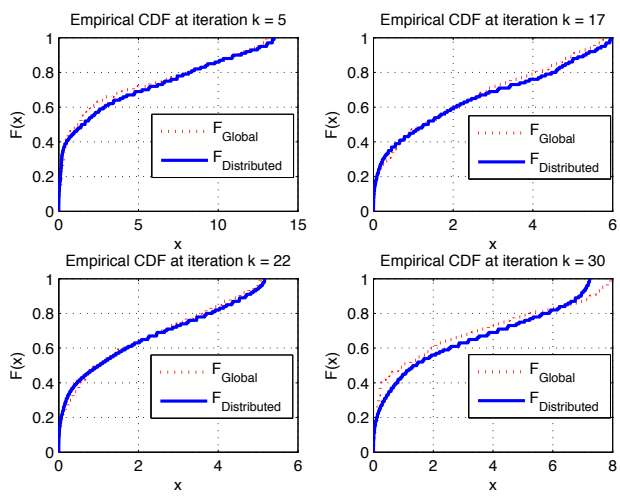

Fig. 4. CDFs for the $X$-coordinate of the target estimated using the centralized UPF and CD/UPF for $k=5,17,22,30$.

non-linear systems. In our simulations, the tracking performance of the CD/UPF is almost identical to the centralized particle filter. As future work, we intend to focus on the convergence properties of the concensus step of the $\mathrm{CD} / \mathrm{UPF}$.

\section{REFERENCES}

[1] X. Sheng, Y. Hu, and P. Ramanathan, "GMM approxim. for multiple targets localization and tracking in WSN," in 4th Int. Sym. on Inf. Process. in Sensor Networks, 2005, pp. 181-188.

[2] R. Olfati-Saber, A. Fax, and R. M. Murry, "Consensus and coopration in networked multi-agent systems," in Proceedings of the IEEE, 2007.

[3] M. J. Coates and B. N. Oreshkin, "Asynchronous distributed particle filter via decentralized evaluation of gaussian products," in Proc. ISIF Int. Conf. Inf. Fusion, 2010.

[4] D. Gu S. Junxi, H. Zhen, and L. Hongzuo, "Consensus based distributed particle filter in sensor networks," in IEEE Int. Conference on Information and Automation, 2008, pp. 302-307.

[5] A.G. Dimakis, S. Kar, J.M.F. Moura, M.G. Rabbat and A. Scaglione, "Gossip algorithms for distributed signal processing," Proc. of the IEEE, vol. 98, pp. 1847-1864, 2010.

[6] R. Van der Merwe, A. Doucet, N. de Freitas, and E. Wan, "The unscented particle filter," Tech. Rep. CUED/F-INFENG/TR 380, Cambridge University, 2000.

[7] M. Arulampalam, B. Ristic, N. Gordon, and T. Mansell, "Bearings only tracking of maneuvering targets w/ particle filters," EURASIP Appl. Sig. Process., vol. 15, pp. 2351-65, 2004.

[8] Y. M.Zhu, Z. S. You, J. Zhao, K.-S. Zhang, and X. R. Li “The optimality for the distributed Kalman filtering fusion with feedback," Automatica, vol. 37, pp. 14891493, 2001. 